\title{
A case based reasoning architecture and component based model for Green IS implementation and diffusion in organisation
}

\begin{abstract}
Green Information Systems (IS) offers promise for IS scholars to make significant contribution in reducing greenhouse gas emissions, achieve sustainability and reducing the effects of global climate change. Green IS has therefore become one of the latest considerations to improve organization's environmental sustainability whilst reducing the cost of IT processes. Due to recent research centered on sustainability, a few number of Green IS models and frameworks have been developed for addressing environmental sustainability, however there is still problem relating to provision of adequate data that can train, educate and support practitioner in diffusing and implementing Green IS in organisations. Thus after reviewing existing research of Green IS, this paper proposed a Case Based Reasoning (CBR) architecture for Green IS Implementation in organisations to train, educate and support practitioner in implementing Green IS in their organisation and a Component Based Model to support Practitioners in diffusing and achieving EcoSustainability goal. Contribution from this paper can also be used as a guide for future research in this domain
\end{abstract}

Keyword: Case based reasoning; Green IS; Green IT; Sustainability; Green IS components 